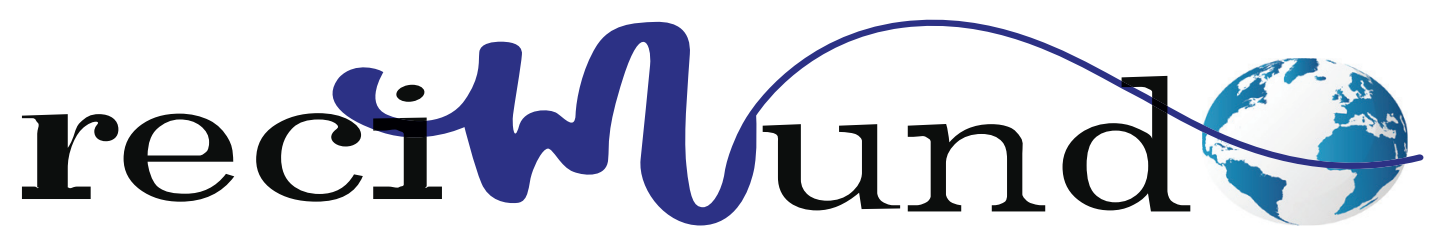

Revista Científica Mundo de la Investigación y el Conocimiento

DOI: 10.26820/recimundo/4.(1).enero.2020.162-172

URL: http://recimundo.com/index.php/es/article/view/738

EDITORIAL: Saberes del Conocimiento

REVISTA: RECIMUNDO

ISSN: 2588-073X

TIPO DE INVESTIGACIÓN: Artículo de Revisión

CÓDIGO UNESCO: 3201.10 Pediatría

PAGINAS: $162-172$

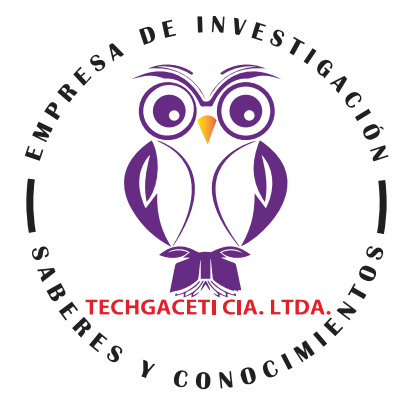

\title{
Terapia de hidratación parenteral en pediatría
}

\section{Parenteral hydration therapy in pediatrics}

\section{Terapia de hidratação parenteral em pediatria}

Jessenia Katiuska Pilay Ponce1; Stefanie Cristina Reinoso Tapia2; Andrés Alberto Vásquez Gaibor³;

Diego armando Dávila Peralta ${ }^{4}$

\section{RECIBIDO: 18/09/2019 ACEPTADO: 29/10/2019 PUBLICADO: 31/01/2020}

1. Médico; Investigador Independiente; Guayaquil, Ecuador; katiuska_pilay85@hotmail.com; (iD https://orcid. org/0000-0002-8418-8623

2. Médico; Investigador Independiente; Guayaquil, Ecuador; stefaniee@hotmail.es; (DD https://orcid.org/00000001-6394-5202

3. Médico; Investigador Independiente; Guayaquil, Ecuador, Ecuador; andresvq3@hotmail.com; (DD https://orcid. org/0000-0002-9536-3423

4. Médico; Investigador Independiente; Guayaquil, Ecuador; Idieevil14@hotmail.com; (iD https://orcid.org/00000002-0086-8403

CORRESPONDENCIA

essenia Katiuska Pilay Ponce

katiuska_pilay85@hotmail.com

Guayaquil, Ecuador 


\section{RESUMEN}

La deshidratación en el paciente pediátrico es una de las causas de morbimortalidad más importantes en países en vía de desarrollo, de allí que sea una de las principales patologías que se atienden en los servicios de urgencia pediátricos. Es importante considerar que el cuerpo de un niño está principalmente constituido por agua, de tal forma que cualquier patología puede comprometer algunos de los principales órganos e incluso los sistemas circulatorio, renal, nervioso, respiratorio y digestivo, y causar complicaciones que pudieran prevenirse con el adecuado y oportuno tratamiento.

El tratamiento parenteral representa una de las formas más eficaces de tratar la deshidratación por la rapidez en cuanto a la vía de administración aunado a la composición de las ampollas. En tal sentido, el presente estudio tiene el objeto de discernir sobre el tratamiento parenteral y su uso en pediatría.

Palabras clave: Pediatría, Parenteral, Deshidratación, Tratamientos, Hidrotratamientos, Rehidratación, Inyecciones, Vía Intravenosa, Fluidos, Diarrea.

\section{ABSTRACT}

Dehydration in the pediatric patient is one of the most important causes of morbidity and mortality in developing countries, hence it is one of the main pathologies treated in pediatric emergency services. It is important to consider that the body of a child is mainly constituted by water, so that any pathology can compromise some of the main organs and even the circulatory, renal, nervous, respiratory and digestive systems, and cause complications that could be prevented with the adequate and timely treatment.

Parenteral treatment represents one of the most effective ways to treat dehydration due to the rapidity of the route of administration combined with the composition of the blisters. In this regard, the present study aims to discern about parenteral treatment and its use in pediatrics.

Keywords: Pediatrics, Parenteral, Dehydration, Treatments, Hydrotreatments, Rehydration, Injections, Intravenous Route, Fluids, Diarrhea.

\section{RESUMO}

A desidratação no paciente pediátrico é uma das causas mais importantes de morbimortalidade nos países em desenvolvimento, portanto, é uma das principais patologias tratadas em serviços de emergência pediátrica. É importante considerar que o corpo de uma criança é constituído principalmente por água, para que qualquer patologia possa comprometer alguns dos principais órgãos e até os sistemas circulatório, renal, nervoso, respiratório e digestivo, além de causar complicações que poderiam ser evitadas com a tratamento adequado e oportuno.

O tratamento parenteral representa uma das maneiras mais eficazes de tratar a desidratação devido à rapidez da via de administração combinada com a composição dos blisters. Nesse sentido, o presente estudo objetiva discernir sobre o tratamento parenteral e seu uso em pediatria.

Palavras-chave: Pediatria, Parenteral, Desidratação, Tratamentos, Hidrotratamentos, Reidratação, Injeções, Via Intravenosa, Fluidos, Diarréia. 


\section{Introducción}

La deshidratación en adultos puede comprometer algunos de los principales órganos e incluso los sistemas circulatorio, renal, nervioso, respiratorio y digestivo; en los niños es aún más delicado. En cualquiera de los casos, esta dificultad dependerá del grado de pérdida de líquidos que pueda presentar el paciente. De allí la importancia de conocer sobre los tratamientos de hidratación existentes, específicamente para los infantes, su uso y sus formas de aplicación; así como, distinguir los diferentes grados de deshidratación y los tratamientos acordes a cada caso.

El contenido hídrico del organismo varía en proporción inversa a la cantidad de grasa del cuerpo, en efecto, los infantes tienen mayor porcentaje de agua. El cuerpo humano está principalmente constituido por agua, en adultos representa el $60 \%$ al menos del peso corporal, en los niños alcanza $70 \%$ y en los recién nacidos puede llegar a alcanzar los $80 \%$.

Diversas patologías conducen a la deshidratación, de allí que ésta sea una de las causas más comunes de consulta en el servicio de urgencias de pediatría. El manejo inadecuado de los tratamientos para revertir la deshidratación puede conducir a los pacientes a complicaciones previsibles como:

- Shock hipovolémico.

- Falla renal.

- Alte-rraciones del estado de conciencia e incluso la muerte.

De tal manera que es menester un abordaje integral al paciente con este estado, para identificar cual es el cuadro clínico y así poder detectar la causa origen de la deshidratación, en aras de establecer el tratamiento el adecuado para la rehidratación y reconstitución del estado de salud del mismo.

En cuanto a la deshidratación en el pacien- te pediátrico es importante acotar, que ésta es reconocida como una de las causas de morbimortalidad más recurrente en países en vía de desarrollo; por tanto, es imprescindible el correcto manejo de esta patología con el fin de disminuir los desenlaces fatales y prevenibles de la enfermedad

Uno de los tratamientos comúnmente adoptados por su eficacia y rápida es la hidratación Parenteral, que como su nombre lo índica, se refiere a "parenteral" se a la vía de administración de los fármacos, ya que se atraviesa una o más capas de la piel o de las membranas mucosas mediante una inyección por la vía intravenosa.

El presente estudio se propone dar a conocer las principales causas, la fisiopatología, la clínica y el tratamiento más adecuado según la evidencia actual de la deshidratación en el paciente pediátrico con el fin de en aras de disminuir las probabilidades de posibles complicaciones de esta patología.

\section{Método}

En función de alcanzar el objetivo planteado por el presente estudio, se ha llevado a cabo la metodología de investigación documental, revisando más de 50 fuentes bibliográficas, y analizándolas bajo los criterios de inducción e inferencias, en tono a las los planteamientos más comunes respecto a las Deshidratación en niños, así como planteamientos encontrados referente a tratamientos parenteraly su uso pediátrico con el propósito de aportar nuevos conocimientos de relevancia para evitar los efectos que pueden ocasionarse por la deshidratación.

Libros de carácter científico, documentos electrónicos, sitios web que dedican su portal tanto a los pacientes como especialistas y artículos de revistas científicas, garantizan la heterogeneidad de los hallazgos teóricos y prácticos analizados en el presente estudio. Se consideraron los aportes más relevantes de especialistas y los planteamientos teóricos de éstos para resaltar ideas consensuadas y plasmar las disyuntivas 
que se establecen en torno a la fisiopatología, fisiología, la clínica, las indicaciones y las contraindicaciones de las sales de rehidratación oral y las solu $\neg$ ciones de administración endovenosa, para futuras investigaciones y casos de estudio.

\section{Deshidratación - Causas y Factores:}

En al menos en 17 países de América Latina las enfermedades diarreicas se encuentran entre las primeras cinco enfermedades que causan de muerte por complicaciones. Aún y cuando más del $90 \%$ de pacientes suelen responder al tratamiento sintomático, debe prevenirse dentro del abordaje terapéutico las posibles complicaciones. Al respecto (Ochoa, Posada, Restrepo, \& Aristizabal, 2008) manifiestan: "La desnutrición es frecuente y los niños desnutridos tienen mayores posibilidades de morir en un episodio diarreico, que los que están bien nutridos. Además, los lactantes y niños menores desarrollan deshidratación más rápidamente que los niños mayores o los adultos".

1. Factores Clínicos: Entre los factores clínicos y epidemiológicos asociados a la deshidratación en niños podemos mencionar el déficit nutricional, presencia de vómitos, ausencia de electrolítico y otros. Dichos factores deben conocerse para potenciar los tratamientos preventivos y curativos; entre estos, se pueden referir:

- Vómitos.

- Deposiciones.

- Presencia de Fiebre.

- Ausencia de Rehidratación oral.

- Privación de antibioticoterapia.

- Antecedente de personal de disentería.

- Lactante.

2. Factores socio económicos: Estos factores aumentan la vulnerabilidad del infante. En efecto, existen factores que pueden predisponer al paciente a un posible cuadro de deshidratación, y los niños son más vulnerables (Molina Rivera, 2016). Entre estos, se tienen :

- Hacinamiento, falta de acceso al agua potable, falta de posibilidades de refrigeración de los alimentos, sistema de eliminación de excretas ineficiente.

- Falta de acceso a información.

- Dificultad de acceso a los servicios de salud.

- Dificultad para aplicar los cuidados necesarios al paciente.

- Analfabetismo, desocupación.

3. Factores del huésped

- Niños menores de un año.

- Falta de lactancia materna exclusiva durante los primeros 6 meses de vida.

- Uso de biberones.

- Desnutrición.

- Inmunosupresión.

La causa más común de la aparición de deshidratación es la enfermedad diarreica. Durante un episodio de diarrea,se pierde agua y electrolitos (sodio, cloruro, potasio y bicarbonato) en las heces líquidas, los vómitos, el sudor, la orina y la respiración; a consecuencia de todo esto ocurre ladeshidratación. (Organización Mundial de la Salud, 2007)

Entre los grados de deshidratación y sus sintomatologías, se clasifican en:

1. Deshidratación incipiente: Sin signos ni síntomas.

2. Deshidratación moderada:

- Sed.

- Comportamiento inquieto o irritable.

- Reducción de la elasticidad de la piel.

- Ojos hundidos. 
3. Deshidratación grave:

- Los síntomas se agravan.

- Choque, con pérdida parcial del conocimiento, falta de diuresis.
- Extremidades frías y húmedas, pulso rápido y débil, tensión arterial baja o no detectable y palidez.

Tabla 1. Grado de deshidratación según la edad del niño

\begin{tabular}{|c|c|c|}
\hline Deshidratación & Lactante & Niño mayor \\
\hline Leve & $<5 \%$ & $<3 \%$ \\
Moderada & $5-10 \%$ & $3-7 \%$ \\
Severa & $>10 \%$ & $>7 \%$ \\
\hline
\end{tabular}

Fuente: (Cubero Millán \& Machado Casas, 2013)

Una de las enfermedades que origina la deshidratación, incluso no sintomática en su primer estadio, es la Diarrea Aguda (DEA), la cual, es una de las principales causas de morbi mortalidad en niños menores de 5 años. Dicha enfermedad progresivamente acentúa la deshidratación hasta llegar a desarrollar choque hipovolémico con alteración de la conciencia, oliguria, hipotensión, cianosis periférica y de no iniciarse la rehidratación, existe potencial riesgo de que ocurra la muerte.

"Es uno de los principales problemas que afectan a la población infantil generando así una importante demanda de servicios de salud. Estimado que 7 de cada diez niños menores de 5 años han sufrido esta patología." (Quevedo, 2002)

En efecto, la Diarrea Aguda, definida como el estado clínico consecutivo de la pérdida de líquidos y solutos en el cuerpo humano, es un desequilibrio ácido-base del organismo que puede generar un compromiso en las funcionescorporales.

En un estudio realizado en el Hospital de San José de Lima (Molina Rivera, 2016), planteo que la DEA, en su hallazos el 75 a $80 \%$ de los casos compromete a lactantes menores de 12 meses ymás del 90\% por debajo de los 18 meses. Y clasifica esta patología por el tiempo deduración:

- Aguda (menos de 14 días).
- Prolongada o persistente (más de 14 días).

Diagnostico de la deshidratación y sus grados:

Todo niño que acude a un servicio de salud y consulta debe ser evaluado de forma cuidados a mediante una anamnesis minuciosa y la exploración física minuciosa, los cuales deben estar dirigidos -aunque no en forma exclusiva- a la Enfermedad Diarreica Aguda. En la gran mayoría de los casos se requiere la valoración del paciente a la luz de la información clínica. A continuación se describe información útil en el niño con EDA, especialmente para clasificar su severidad, decidir el manejo y en lo posible aclarar su diagnóstico:

- Número de días de evolución de la diarrea.

- Presencia de sangre en las heces.

- Cantidad y calidad de la reposición que se ha hecho de las pérdidas.

- Presencia de brotes locales de cólera.

- Tratamiento reciente con antibiótico y otro medicamento.

- En el lactante, ataque de llanto con palidez.

- Alimentación del niño. 
Tabla 2. Clasificación de la Deshidratación en niños con Diarrea

\begin{tabular}{|l|l|l|l|}
\hline \multicolumn{1}{|c|}{ Signo } & \multicolumn{1}{|c|}{$\begin{array}{c}\text { No } \\
\text { deshidratación }\end{array}$} & $\begin{array}{c}\text { Alguna deshidratación } \\
\text { Si el paciente tiene dos o más } \\
\text { signos, incluyendo un signo }\end{array}$ & \multicolumn{1}{|c|}{$\begin{array}{c}\text { Deshidratación severa } \\
\text { Si el paciente tiene dos o más } \\
\text { signos, incluyendo un signo }\end{array}$} \\
\hline Condición general $^{1}$ & Normal, alerta & Agitado, irritable & Letárgico o inconsciente \\
\hline Ojos $^{1}$ & Normales & Hundidos & Muy hundidos y secos \\
\hline Lágrimas & Presentes & Ausentes & Ausentes \\
\hline Boca y lengua ${ }^{2}$ & Húmedas & Secas & Muy secas \\
\hline Ojos & Normales & Normal o hundidos & Hundidos \\
\hline Sed & No sed, bebe & Sediento, bebe con avidez & Bebe poco o no puede beber \\
\hline Pliegue cutáneo ${ }^{3}$ & Regresa rápido & Regresa lentamente & Regresa muy lentamente \\
\hline
\end{tabular}

Fuente: (Organización Mundial de la Salud, 2007)

\section{Tipos de Deshidratación}

Clasificación de la deshidratación según la tonicidad:

- Hipotónica: natremia inferior a 130 mE$\mathrm{q} / \mathrm{l}$.

- Isotónica: natremia entre 130 a 150 mE$\mathrm{q} / \mathrm{l}$.

- Hipertónica: natremia superior a 150 $\mathrm{mEq} / \mathrm{l}$.

Déficit de sodio según los tiposde deshidratación:

- Hipotónica: 10 a 14 mEq/kg/día.

- Isotónica: 7 a 10 mEq/kg/día.

- Hipertónica: 2 a 4 mEq/kg/día.

\section{Hidratación en Pediatría}

La rehidratación oral es el método de elección para el tratamiento de las deshidrataciones leves y moderadas debidas a la diarrea.Mientras que en la deshidratación grave se administra $20 \mathrm{cc} / \mathrm{kg}$ de SSI (solución salina isotónica al 0,9\%) o Ringer Lactato, en goteo rápido, con posterior valoración clínica para eventuales repeticiones de la misma solución.

Una vez superada la situación de shock, se procede a la re-hidratación calculando el déficit del paciente, para lo cual se debe sumar el volumen de mantenimiento más el porcentaje de pérdida a reponer en 24 horas.
La hidratación endovenosa en deshidratación por diarrea se indican en los siguientes cuadros:

1. Insuficiencia circulatoria con shock hipovolémico.

2. Deshidratación grave (más del $10 \%$ de pérdida de peso).

3. Hiponatremia severa $([\mathrm{Na}+]<120 \mathrm{mE}-$ $\mathrm{q} / \mathrm{L})$ o hipernatremia severa $([\mathrm{Na}+]>$ $160 \mathrm{mEq} / \mathrm{L})$.

4. Fracaso de hidratación oral: vómitos persistentes (más de 4 vómitos por hora) distensión abdominal o íleo paralítico.

5. Alteración del estado de conciencia o convulsiones durante la hidratación oral.

6. Pérdidas gastrointestinales de tan alto volumen y frecuencia que no puedan ser restituidas por la ingesta de suero oral (volumen fecal mayor de 20-30 cc / $\mathrm{kg} /$ hora).

7. Deterioro de su cuadro clínico o ausencia de mejoría clínica a las 8 horas de haber iniciado la vía oral.

\section{Vías de Administración}

Establecer un acceso vascular es vital para la administración de líquidos al paciente con compromiso circulatorio, en el caso de los infantes, requiere de mayor atención y debe situarse en el lugar que permita el acceso vascular de forma sencilla.

Entre estas podemos mencionar: El catete-

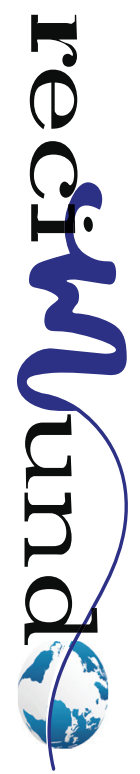


rismo venoso, la vía intraósea, el cateterismo venoso central y la flebotomía

El cateterismo venoso periférico es el método de acceso vascular de elección en pacientes con deshidratación. Se recomiendan catéteres del mayor calibre posible para permitir la administración rápida de un gran volumen de líquidos. Las venas periféricas más adecuadas, por su mayor tamaño y localización anatómica, son la vena cubital mediana del codo y la safena interna en el tobillo. Sin embargo, en los infantes con deshidratación grave, puede ser técnicamente difícil y toma mucho tiempo.

En caso de no lograr un acceso venoso periférico rápido, se considera que la vía intraósea es la mejor alternativa para obtener un acceso vascular, debido a la facilidad, rapidez y seguridad de la técnica. Es importante acotar que la cavidad medular de los huesos largos está ocupada por una rica red de capilares sinusoides que drenan a un gran seno venoso central, el cual no se colapsa ni siquiera en situación de shock y permite pasar los fármacos y líquidos a la circulación general con una rapidez, aproximadamente de 30 a 60 segundos, en un 98\% de los casos. El sitio anatómico recomendado para la infusión intraósea en recién nacidos, lactantes y menores de 6 años es el segmento proximal de la tibia (1 a $3 \mathrm{~cm}$ por debajo de la tuberosidad tibial en la superficie antero interna), mientras que en mayores de 6 años y adolescentes es el segmento distal de la tibia ( 1 a 2 $\mathrm{cm}$ por encima del maléolo interno). Cabe mencionar los dispositivos automáticos de inserción de agujas intraóseas, como la pistola de inyección intraóseay el sistema de inyección intraóseo EZ-IO, que ofrecen un acceso rápido y seguro al sistema vascular para todas las edades. Su uso se recomienda, en especial, para el acceso a nivel de la tibia y en niños mayores de 6 años, adolescentes y adultos.

Otra alternativa para el acceso vascular es el cateterismo venoso central, el cual brinda una vía segura y estable, que además permite controlar la presión venosa central. En caso de ser necesario, se prefiere la vena femoral, por mayor facilidad y seguridad técnica; en su defecto, se utiliza la vena yugular interna y, en infantes mayo-res, la vena subclavia. En recién nacidos, puede utilizarse la vena umbilical.

Las complicaciones del cateterismo venoso central son potencialmente graves (tromboembolismo pulmonar, sangrado venoso o arterial, neumotórax, hidrotórax, hemotórax y arritmias, entre otras); se presentan con mayor frecuencia en lactantes y menores de 2 años, por lo que sólo debe ser realizado por personal con amplia experiencia en la técnica.

Actualmente, la flebotomía es considerada como la última opción para lograr el acceso vascular. Está indicada sólo en casos de falla de todos los otros métodos ya que requiere de un tiempo (promedio de 15-25 minutos).

\section{Hidratación Intravenosa}

La hidratación intravenosa varía dependiendo de la gravedad de la deshidratación (isonatrémica, hiponatrémica o hipernatrémica) y de los déficits de otros iones y trastornos ácido-base.

Se realiza en 2 fases: estabilización y reposicióndel déficit de líquidos y electrolitos, además de las pérdidas concurrentes.

Estabilización: Es menester conocer el shock hipovolémico, que consiste en que a medida que la volemia disminuye, la resistencia vascular aumenta y esto permite conservar la tensión arterial en un nivel normal (shock compensado). Sípor el contrario, continúa la pérdida de volemia sin un reemplazo adecuado, disminuye la tensión arterial y hay un deterioro rápido y progresivo por hipoxia tisular e isquemia, que unidos desencadenan una cascada de eventos que conducen a falla multiórganica y muerte (shock descompensado) 
Por lo tanto, en un paciente pediátrico con signos de deshidratación, asociados con signos de hipoperfusión, tales como alteración del llenado capilar, taquipnea, taquicardia severa, alteración del estado de conciencia, respiración acidótica y ausencia de orina en las últimas 4 horas, aún en presencia de una tensión arterial normal (shock compensado), debe procederse a una rápida reposición del volumen intravascular. La fase de emergencia o restitución de la volemia es similar para cualquier tipo de deshidratación.El volumen a utilizar corresponde al $25 \%$ de la volemia (expansiones de $20 \mathrm{ml} / \mathrm{kg}$ ). Este volumen puede repetirse cada 10 a 15 minutos hasta restablecer la perfusión sanguínea, lo cual se manifiesta con mejoría del estado de conciencia, presencia de diuresis y la normalización de las variables hemodinámicas del paciente
El volumen máximo total a administrar en la fase de expansión es de 60 a 80ml/kg en una hora. Si luego de esto no se observa mejoría, deben considerarse otros diagnósticos asociados (insuficiencia cardíaca, sepsis o insuficiencia renal) y pasar al paciente a una unidad de cuidados intensivos para realizar un monitoreo hemodinámico invasivo y administrar tratamientos a base de inotrópicos, vasodilatadores o vasopresores en infusión continua .Una vez restituida la volemia y superada la insuficiencia circulatoria, debe planificarse la hidratación en el paciente para continuar con a rehidratación oral o una deshidratación con déficit de 10\% para hidratación por vía endovenosa.

Tabla 3. Solución intravenosa más utilizadas

\begin{tabular}{lc|c|c|c|c|}
\hline Líquido & $\mathrm{Na}+$ & $\mathrm{Cl}-$ & $\mathrm{K}+$ & $\mathrm{Ca}++$ & Lactato \\
\hline Sol. Salina Isotónica $(0,9 \% \mathrm{ClNa})$ & 154 & 154 & & & \\
$1 / 2 \mathrm{SSI}(0,45 \% \mathrm{ClNa}) 25 \mathrm{cc} \mathrm{ClNa} 3 \mathrm{M}$ & 75 & 75 & & & \\
$1 / 4 \mathrm{SSI}(0,2 \% \mathrm{ClNa}) 12 \mathrm{cc} \mathrm{ClNa} 3 \mathrm{M}$ & 36 & 36 & & & \\
Solución Ringer Lactato & 130 & 109 & 4 & 3 & 28 \\
\hline
\end{tabular}

Fuente: Autores 2020

Segunda fase: de reposición del déficit y aporte de líquidos y electrolitos de mantenimiento y pérdidas concurrentes: Para establecer los esquemas de hidratación el primer paso debe ser calcular las necesidades basales de mantenimiento de líquidos y electrolitos para el paciente. Para el cálculo de las necesidades hídricas y de electrolitos de mantenimiento se utiliza el método del gasto calórico, basado en que las necesidades de agua y de electrolitos se relacionan con más precisión con el gasto de calorías que con el peso corporal .El método más usado es el de Holliday-Segar, con estimaciones de gasto de calorías en categorías de peso fijo, el cual asume que por cada 100 calorías metabolizadas son necesarios $100 \mathrm{ml}$ de H2O. En la práctica, por cada $100 \mathrm{Kcal}$ gastadas se requie- ren unos $50 \mathrm{ml}$ de líquido para la piel, vías respiratorias y las pérdidas de materia fecal basal; son necesarios 55-65 ml de líquido para que los riñones puedan excretar orina no concentrada

\section{Terapia de Hidratación parenteral en Pe- diatría}

Fluidoterapia según los diferentes tipos de deshidratación:

Los esquemas de aportes pueden variar según magnitud de nuevas pérdidas concurrentes y sucesivas determinaciones de electrolitos. Estas recomendaciones se enmarcan en niños con mecanismos homeostáticos normales de excreción urinaria de agua, sodio y potasio.Pacientes con trastornos fisiopatológicos más complejos como

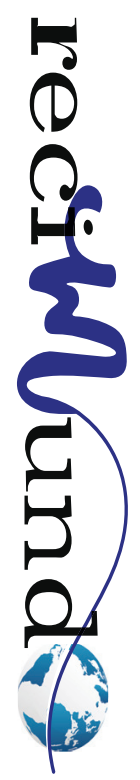


insuficiencia renal, diabetes insípida,etc., deben ser tratados con consideraciones especiales inherentes al trastorno de base.

\section{Deshidratación Isonatrémica(Natremia: entre 130-150 mEq/l)}

- Si el niño se halla en shock, se procede a la reposición del volumen intravascular con SSI a razón de 20 ml/kg en 20 minutos, que eventualmente se repite hasta restablecer la hemodinámica.

- Restauración del déficit en 24 horas, aportando 7 a $10 \mathrm{mEq} / \mathrm{kg} /$ día de sodio. Este aporte se consigue con una concentración de 60 mEq/litro.

- El potasio se agrega a la mezcla una vez que la diuresis se restituye, en forma de cloruro de potasio a una concentración de $30 \mathrm{mEq} / \mathrm{litro}$ del mencionado catión.

\section{Deshidratación hiponatrémica (Natremia: inferior a130 $\mathrm{mEq} / \mathrm{l}$ )}

- Si el niño se halla en shock, se procede a la reposición del volumen intravascular con SSI a razón de 20 ml/kg en 20 minutos, que eventualmente se repite hasta restablecer la hemodinamia

- Restauración del déficit en 24 horas, la mitad del mismo en las primeras 8 horas, y el resto en las 16 horas restantes, aportando 10 a 14 mEq/kg/día de sodio.

- Este aporte se consigue con una concentración de sodio de $75 \mathrm{mEq} / \mathrm{litro}$, en las primeras 8 horas y de $60 \mathrm{mEq} / \mathrm{l}$ en las restantes 16 horas.

- La corrección muy rápida de la hiponatremia puede conducir a la mielinólisispontina, por lo que se debe evitar un incremento del sodio sérico mayor de 12 $\mathrm{mEq} / \mathrm{l}$ en 24 horas.

- La corrección del déficit de sodio se puede cuantificar por la siguiente fórmula (Na deseado - Na actual) x 0.6 x Kg.

- El factor 0.6 corresponde a la distribu- ción del sodio en el líquido extracelular.

- Con el potasio se procede de la misma manera que en la deshidratación isonatrémica.

\section{Deshidratación hipernatrémica (Natre- mia: superior a $150 \mathrm{mEq} / \mathrm{l}$ )}

- Si el niño se halla en shock, se procede a la reposición del volumen intravascular con SSI a razón de 20 ml/kg en 20 minutos, que eventualmente se repite hasta restablecer la hemodinamia.

- La restauración del déficit se realiza en 1 a 4 días según la natremia obtenida:

- Na de 145 a 157 mEq/l: en 24 horas.

- Na de 158 a 170 mEq/l: en 48 horas.

- Na de 171 a 183 mEq/l: en 72 horas.

- Na de 184 a 194 mEq/l: en 84 horas.

- La reposición de cada día contempla el mantenimiento más el déficit estimado, que se fraccionara en 1 a 4 días según el tiempo determinado por el nivel de natremia.

- No existe un consenso sobre la concentración ideal del líquido a infundir, las recomendaciones del plan inicial varían de $36 \mathrm{mEq} / \mathrm{litro}(1 / 4 \mathrm{de} \mathrm{SSI})$ a $75 \mathrm{mEq} /(1 / 2 \mathrm{SSI})$. Se deben variar las concentraciones de sodio según la velocidad de descenso del sodio, lo cual esta directamente relacionado con el aporte de "agua libre". En pacientes complicados con hipernatremia grave, se infunden "en paralelo" 2 soluciones con diferentes concentraciones de sodio y la misma concentración de glucosa y potasio:

a) Solución glucosada al 5\% + 36 $\mathrm{mEq} / \mathrm{l}$ de sodio ( $1 / 4$ de SSI).

b) Solución glucosada al 5\% + 150 $\mathrm{mEq} / \mathrm{l}$ de sodio (SSI). 
Déficit de potasio según los tipos de deshidratación

- Hipotónica: 8 a 10 mEq/kg/día.

- Isotónica: 8 a 10 mEq/kg/día.

- Hipertónica: 0 a 4 mEq/kg/día.

\section{Consideraciones generales en el trata- miento parenteral:}

La restitución del potasio, se debe considerar las siguientes recomendaciones:

- No debe agregarse a la mezcla de hidratación hasta que el paciente no haya orinado y se compruebe la buena funcionalidad de los riñones.

- La corrección de la kalemia, a diferencia del sodio, se realiza de manera gradual en 48 horas o más, motivo por el cual las concentraciones indicadas en la mezcla de rehidratación equivalen a la mitad o menos con respecto al sodio, a pesar que los déficits sean similares.

- El aporte global de potasio por día no debe exceder de $4 \mathrm{mEq} / \mathrm{día}$, para evitar sobrepasar la capacidad de captación celular de potasio, y por consiguiente la hiperpotasemia.

- El nivel sérico de potasio se ve alterado en forma inversamente proporcional por los desequilibrios del estado acido básico. Así, por cada 0,1 que desciende el $\mathrm{pH}$, el nivel de potasio se incrementa entre 0,6 mEq/l. En el curso de la re-hidratación la acidosis tiende a corregirse espontáneamente, y el potasio desciende consecuentemente, de ahí la importancia de los controles sucesivos del ionograma.

\section{Líquidos de reposición}

En el curso de la rehidratación pueden continuar las pérdidas concurrentes, las cuales deben ser tenidas en cuenta según el sitio del débito.
Líquido gástrico: ya sea por vómitos o drenaje por sonda, la reposición se debe hacer teniendo en cuenta la composición del mismo y el volumen perdido:

- Sodio $=20-80 \mathrm{mEq} / \mathrm{l}$.

- Potasio $=5-20 \mathrm{mEq} / \mathrm{l}$.

- Cloruro $=100-150 \mathrm{mEq} / \mathrm{l}$.

Una solución adecuada para la reposición, volumen por volumen, en un tiempo de 1 a 6 horas, es:SSI hasta $1000 \mathrm{cc}+7 \mathrm{ml}$ de $\mathrm{Cl}$ $\mathrm{K}$, que representa una cantidad mayor de potasio que la que se pierde, teniendo en cuenta la tendencia a desarrollar hipokalemia en pacientes con pérdidas gástricas, debido a las pérdidas concomitantes por orina.

Diarrea: en los infrecuentes casos de imposibilidadde reposición por vía oral de pérdidas por deposicionesdiarreicas copiosas, se debe medir el volumende las mismas e infundir la cantidad equivalente segúnla composición de la pérdida:

- Sodio = 10 a $90 \mathrm{mEq} / \mathrm{l}$.

- Potasio $=10$ a $80 \mathrm{mEq} / \mathrm{l}$.

- Cloro $=10$ a $110 \mathrm{mEq} / \mathrm{l}$.

- Bicarbonato = 15 a $50 \mathrm{mEq} / \mathrm{l}$.

La solución recomendada para la reposición es:

- Solución glucosada al $5 \%$ hasta $1000 \mathrm{cc}$ + $25 \mathrm{mlde} \mathrm{Cl} \mathrm{Na}(75 \mathrm{mEq} / \mathrm{l}$ de sodio) + 20 $\mathrm{ml}$ de Bicarbonato de Sodio 1M (20 mE$\mathrm{q} / \mathrm{l})+8 \mathrm{ml}$ de Cl K (24 mEq/l de Potasio).

Fiebre: por cada grado centígrado que aumenta la temperatura por encima de $37^{\circ}$, se debe calcular $10 \mathrm{ml} / \mathrm{kg} / \mathrm{día}$, aunque este razonamiento es teórico ya que la elevación térmica no persiste durante todo el día, por lo que se debe hacer balances de pérdidas parciales. 


\section{Conclusiones}

- La deshidratación y sus consecuencias pueden ser prevenidas en el caso de los niños y debe ser tratada de forma eficaz. El tratamiento parenteral constituye la vía masídonea y rápida para atender deshidratación agudas.

- El diagnóstico inmediato y la identificación del tipo de deshidratación permitirá emplear el tratamiento mas adecuado según el caso reportado del infante.

- El personal especializado debe estar capacitado para la aplicación oportuna del tratamiento, y manejar los avances tecnológicos para su aplicación.

\section{Bibliografía}

Botella Dorta, C. (15 de Septiembre de 2009). Fisterra. Recuperado el Enero de 2020, de https:// www.fisterra.com/material/tecnicas/parenteral/AdmonParent1.pdf

Calvo, v., \& Planas, M. (2008). Interrelación entre fármacos y nutrientes en situaciones fisiopatológicas determinadas. Barcelona España: Glosa.

Carranza, C., Gómez, J., \& Wilches, L. (2016). Rehidratación en el Paciente Pediátrico. Revista Med, 24(2), 33-46.

Cubero Millán, M. I., \& Machado Casas, I. S. (2013). Deshidratación aguda en pediatría. Actualización del protocolo de rehidratación en el SUE. Granada.
Doldán Pérez, O. (2009). Hidratación Parenteral en Pediatría. Pediatría (Asunción), 36(2), 138-143.

Molina Rivera, S. (2016). FACTORES ASOCIADOS A DESHIDRATACIÓN EN NIÑOS MENORES DE 5 AÑOS CON ENFERMEDAD DIARREICA AGUDA ATENDIDOS EN EL HOSPITAL SAN JOSÉ 20132015. Lima Perú.

Ochoa, L., Posada , R., Restrepo, F., \& Aristizabal, P. (2008). Atención integradas a las enfermedades prevalentes de la infancia. Colombia: Ministerio de Salud de Colombia.

Organización Mundial de la Salud. (2007). Guía práctica: Vigilancia epidemiológica de diarreas causadas por rotavirus. Washington DC.

Potin, M. (2010). Hidrataciön Parental. En M. Potin, \& I. Concha, El niño hospitalizado: problemas frecuentes (pág. 245). Santiago de Chile: Escuela de Medicina de la Pontificia Universidad de Chile.

Quevedo, L. (2002). El pediatra eficiente. Bogotá Colombia: Panamericana.

Rojas G, P. (s.f.). Pontificia Universidad Católica de Chile - Escuela de Medicina. Recuperado el Febrero de 2020, de https://medicina.uc.cl/publicacion/en-diarreas-infantiles-hidratacion-oral-o-endovenosal

Rojas Marcano, I., Castro, M. J., Chacón , L., De Pablos, J. F., López, N., \& Urbina Medina, U. (2009). Hidratación Parenteral. Archivos Venezolanos de Puericultura y Pediatría, 72(4), 154-162.

Urbina Medina, H., Lunar Solé, I., Viscaíno Tenías, R., Sanchez , M., \& Rosario, C. L. (2014). Hidratación Parenteral en Diarrea Aguda. Archivos Venezolados de Puericultura y Pediatría, 77(2), 87-92.

\section{CITAR ESTE ARTICULO:}

Pilay Ponce, J., Reinoso Tapia, S., Vásquez Gaibor, A., \& Dávila Peralta, D. (2020). Terapia de hidratación parenteral en pediatría. RECIMUNDO, 4(1), 162-172. doi:10.26820/recimundo/4.(1).enero.2020.162-172

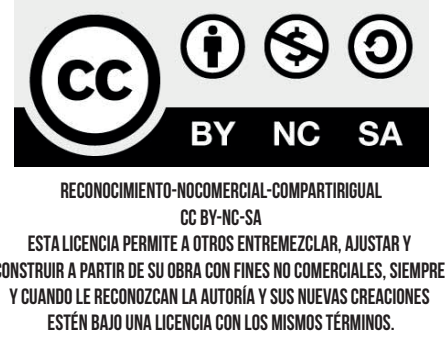

